\title{
RESEARCH ON REMOTE MONITORING OF SMART POWER GRID USING WIRELESS SENSOR NETWORK
}

\author{
Lijuan Cheng ${ }^{1}$ \\ ${ }^{1}$ School of Control Engineering, Xinjiang Institute of Engineering, Urumqi, Xinjiang 830000, China \\ Email:j5175f@163.com
}

\begin{abstract}
For the expanding power grid, its security has been more and more important. Smart grid can monitor the operation status of the power grid using sensors, timely feedback faults, and improve the security of power grid. This paper briefly introduced the ZigBee wireless communication technology and power grid monitoring system based on ZigBee wireless sensor network. The power grid monitoring system was tested in a small-scale local substation and compared with a WiFi based power grid monitoring system. The test items included the detection accuracy of power grid fault and the packet loss rate and time delay of wireless sensor network in the monitoring system. The results showed that the monitoring system designed in this study had higher fault detection accuracy, lower average packet loss rate and maximum packet loss rate, smaller average time delay and maximum time delay and smaller measurement error compared with the WiFi based monitoring system. In conclusion, the grid monitoring based on ZigBee wireless sensor technology can effectively monitor grid.
\end{abstract}

Keywords: Power Grid, Monitoring, Wireless Sensor Network, ZigBee.

\section{Introduction}

Although the scale of power grid and the increase of power generation has meet the demand for electricity, it also increases the risk of failure [1]. In the modern social production which has a extreme demand for electricity, the failure of grid will cause considerable losses [2]. The monitoring of power grid has become an important part of maintaining the security and stability of power grid. Smart grid collects the working information of power grid using sensors and judges the working state of power grid in the server of the monitoring center [3]. The emergence of wireless communication technology makes sensor constitute wireless sensor network, and the transmission of data does not need to rely on data lines such as optical fiber, saving more costs. In addition, after the wireless communication module is integrated into the sensor, it can be more flexible during installation [4]. Sana et al. [5] conducted an in-depth experimental research on Link Quality Estimators (LQEs) which were representative in smart grid distribution substation, and the results showed that the combined LQE was more reliable and stable than the single LQE. Otoum [6] proposed a hierarchical trust based wireless sensor network monitoring model for smart grid equipment and found that the system achieved stability in the early stage and the number of reclustering operations was the least when the threshold value of packet loss probability was 50\%. Grilo et al. [7] proposed a solution to obtain relevant data from selected points in low-voltage power grid and transmit the data to intelligent controller effectively through wireless sensor network and verified the effectiveness of the method. This paper briefly introduced the ZigBee wireless communication technology and power grid monitoring system based on ZigBee wireless sensor network, then tested the power grid monitoring system in a small-scale local substation, and compared it with another WiFi based power grid monitoring system.

\section{Zigbee Based Wireless Communication Technology}

ZigBee wireless communication technology belongs to the short-distance wireless communication in classification, which has advantages of large network capacity, fast response to network access, low operation energy consumption, and low installation cost [8].

Although the wireless communication technology can initiate data exchange anytime and anywhere, in the actual use process, the wireless signal will gradually decay in the process of transmission. Therefore, in order to ensure the quality of wireless communication, a relay node is needed.

The relay node can be a signal amplifier or a communication node itself, which is used for summarizing data and transmitting the data to the target node; in the process, complex communication nodes will form a wireless communication network. 
In this study, it is a wireless sensor network for monitoring power grid [9].

ZigBee is a short-range wireless communication technology as mentioned above. When building a remote monitoring grid system with ZigBee technology, sensors for collecting grid data will form a large number of terminal nodes, and the terminal nodes will combine with relay nodes to form wireless sensor network. Nodes in ZigBee can form three types of network structures. As shown in Figure 1, the terminal equipment is at the end of the whole network and usually collects grid data by combining with various sensors, which is very simple in function and only has functions of data collection and communication with specific equipment; the router and coordinator are the main stem of the whole network, the router is responsible for providing route of data transmission, and the coordinator is responsible for coordinating data transmission route to ensure the balanced working of the router. There is a coordinator in the star topology [10], and the terminal devices and routers are connected with the coordinator. When communicating, they must be forwarded through the coordinator. The cluster topology can be regarded as an extension of the star topology, and the information collected by the terminal nodes is transmitted to the coordinator by taking the router as the transit. The mesh topology also takes the router node as the transit, but its difference with the cluster topology is that any two router nodes in the network can realize information interaction as long as they are in the wireless communication range of the opposite side.

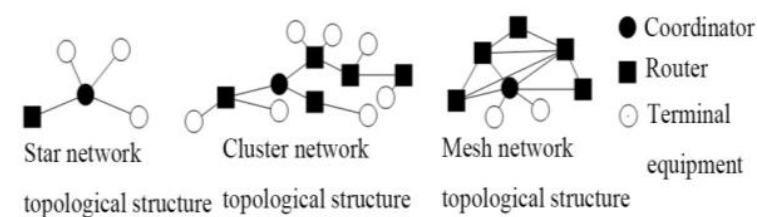

Figure 1: The Topology of Three Kinds of Wireless Sensor Networks

\section{Power Network Monitoring System Based on Wireless Sensor Network}

The working structure of the power grid is shown in Figure 2, in which the power generation unit is mainly various kinds of power plants; the power transformation unit is mainly the transformer, which is used for adjusting the transmission voltage, i.e., up-regulating the voltage when transmitting and adjusting the high voltage to the normal level when arriving at the transmission point; the power transmission unit and distribution unit are mainly composed of wires and cables, which are used for transmitting electric energy; there is a circuit breaker both in the power transmission network and distribution network to ensure that the fault line will be disconnected in time in case of single line fault, so as to ensure the normal operation of the whole power grid. Generally, the flowing power in the transmission network is the high-voltage power.

The power generated by the power station is transmitted in the transmission network after being boosted by the transformer in the substation. Some large factories need the high-voltage power for production work.

Moreover, large factories have their own transformer equipment that can be directly connected to the transmission network. However, general residential users and urban facilities need the transformer to reduce the high-voltage power in the transmission network, and then the power is distributed to different users via power distribution network. It can be said that the transmission network is similar to the trunk of the tree and the distribution network is similar to the branch.

With the expansion of power grid scale, the coverage of power is getting larger and larger, and the voltage in the transmission network is getting higher and higher. Once there is a fault, the loss will be greater. Therefore, the monitoring of power grid is very important.

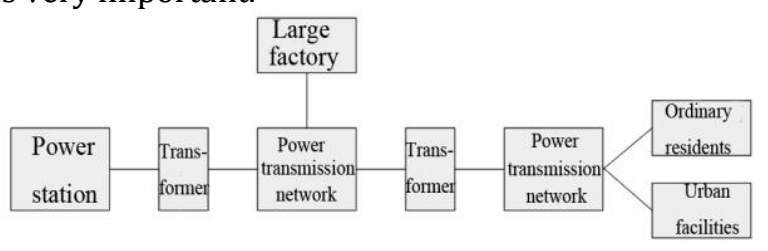

\section{Figure 2: The Working Structure of Power Network}

The monitoring system that can remotely monitor the power grid fault is based on the wireless sensor network.

The basic architecture of the smart grid monitoring system is shown in Figure 3, which can be generally divided into sensors, wireless communication network, servers and monitoring center [12].

Among them, sensors include different kinds of sensors used for detecting the state of power grid, such as current and voltage sensors, temperature and humidity sensors, etc., which are distributed in the monitored power grid as the end nodes of the whole monitoring system; ZigBee module in the wireless communication network is its end node, which is often combined with different kinds of sensors to form the end node of the system in practical application, the module collects data through sensors and sends it to ZigBee routing node, and the routing node finally summarizes the data to the coordinator module [13]; the data collected by ZigBee coordinator module transmits the data to Internet through gateway, and then the monitoring center and server obtain the corresponding grid data through Internet. 


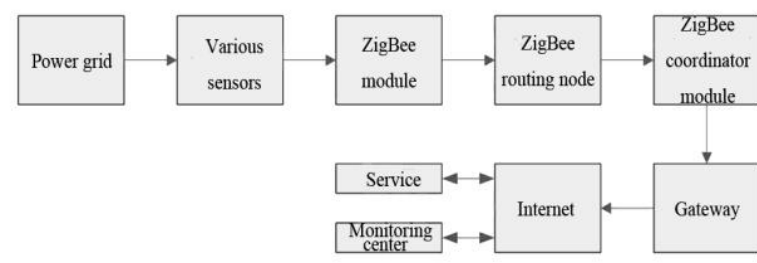

Figure 3: The Basic Structure of Power Grid Monitoring System based on Wireless Sensor Network

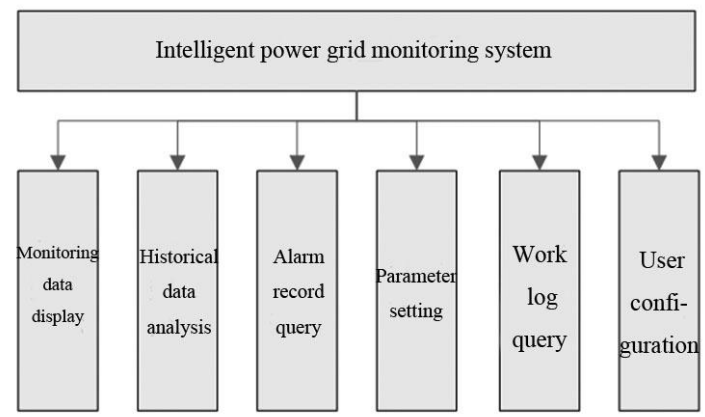

Figure 4: The Functional Structure of the Power Grid Monitoring System based on Wireless Sensor Network

In addition to the above hardware architecture, the corresponding software system is also needed to realize the monitoring of smart grid. The functional structure of the power grid monitoring system based on wireless sensor network is shown in Figure 4.

Monitoring data display function is the real-time display of the power grid data collected by the sensor after wireless transmission. It can assist the monitoring personnel to understand the real-time power grid working conditions and simply judge the working status of power grid and sensor.

Historical data analysis function is to store the data of sensor transmitted by wireless to the server and then draw it into a chart for analysis. Some smart grid monitoring systems even can predict the working state of the grid using intelligent algorithms and historical data, which will not be described in details in this paper.

Alarm record query function is to record the alarm sent by the monitoring system for power grid fault and provide a reference for power grid maintenance and troubleshooting.

Parameter setting function enables users to send instructions to the gateway through the Internet in the monitoring center and transmit them to the sensor through the wireless sensor network to adjust the parameters of the sensor, and to a certain extent, it can also carry out simple maintenance for power grid fault.

Work log query function mainly provides query function for the data of system and power grid operation status, so that users can get effective reference for system and power grid fault repair.

User configuration function is oriented to users, mainly referring to the confirmation of user's identity and authority and the record of common software parameter settings.

\section{Example Analysis 4.1 Experimental environment}

In this study, a small-scale local substation and a power transmission network which was $1 \mathrm{~km}$ less away from the substation were taken as the research subjects, and the effectiveness of the power grid monitoring system based on wireless sensor network was verified by experiments, in which ZigBee wireless communication technology was used in the wireless sensor network.

The main experimental equipment included temperature sensor (model SA1XL-K), closed-loop Hall current sensor (model CHB-150P), closed-loop Hall voltage sensor (model CHV-200), ZigBee module (model CC2530 chip), gateway module (model MGate EIP 3000) and notebook computer (Core i7 processor, 8G memory).

\subsection{Experiment setup}

The test items are as follows.

(1) After the power grid monitoring system based on wireless sensor network was set up in the substation and its nearby transmission line, it operated for four weeks, and the number of times that the system correctly detected the power grid fault during the operation and the actual number of faults obtained with the help of substation staff were recorded to calculate the accuracy of the system.

(2) The wireless sensor network was tested for packet capture to obtain packet loss rate [14]. The number of packets sent and received by the terminal sensor and ZigBee coordinator module were respectively collected through the USB transfer serial port. In this study, the data sending and receiving period between the nodes of wireless sensor network in the power grid monitoring system was $15 \mathrm{~ms}$, the time of sampling using the transfer serial port was $250 \mathrm{~s}$, and the calculation formula of packet loss rate is:

$$
D=1-\frac{N}{M} \text {, }
$$

where $D$ indicates packet loss rate, $N$ represents the number of data packets received by the coordinator module in a cycle, and $M$ indicates the number of data packets sent by the terminal sensor in a cycle.

(3) Test on the data transmission delay of wireless sensor network: the terminal sensor and ZigBee coordinator were sampled using USB transfer serial port. The relevant data collected were the time stamp of the data sent by the terminal sensor and the time stamp of the data received by the coordinator [15]. The time difference between the time stamps was the data delay time. The data sending and receiving period was $15 \mathrm{~ms}$, the sampling time of the transfer serial port was $250 \mathrm{~s}$, and the average delay was calculated according to the time difference of the collected time stamp. 
In order to highlight the effectiveness of ZigBee wireless communication technology in the power grid monitoring system based on wireless sensor network, WiFi communication technology was taken for comparison, the overall structure of the power grid monitoring system was basically the same, ZigBee module which was used for building wireless sensor network was replaced by $\mathrm{WiFi}$ communication module (model USR-WIFI232W600). After replacing the wireless communication module, the above three tests were also carried out.

(4) Test of real-time monitoring data: in the substation, the temperature, current and voltage of the node where the sensor of the monitoring system was located was detected using infrared thermometer, voltage and current transformer, the test time point was recorded, and then the temperature, current and voltage collected by the sensor were retrieved using the monitoring system at the same test time point.

\subsection{Experimental results}

Taking the power grid monitoring system which was based on the wireless sensor network constructed by WiFi technology as a control, the correct number of fault detection of the power grid monitoring system which was based on the wireless sensor network constructed by ZigBee technology was tested, and the results are shown in Figure 5.

The test was carried out for four weeks. With the help of substation staff, the actual failure times of the power grid in four weeks were recorded: five times in the first week, four times in the second week, six times in the third week and seven times in the fourth week.

The times of correctly detected failure of the power grid monitoring system which was based on the wireless sensor network constructed by $\mathrm{WiFi}$ technology were as follows: three times in the first week, one time in the second week, three times in the third week and four times in the fourth week; the times of correctly detected failure of the power grid monitoring system which was based on the wireless sensor network constructed by ZigBee technology in four weeks was consistent with the actual number of failures.

It was seen from Figure 5 that the grid monitoring system which was based on the wireless sensor network constructed by ZigBee technology had higher accuracy in fault detection than the monitoring system using WiFi technology.

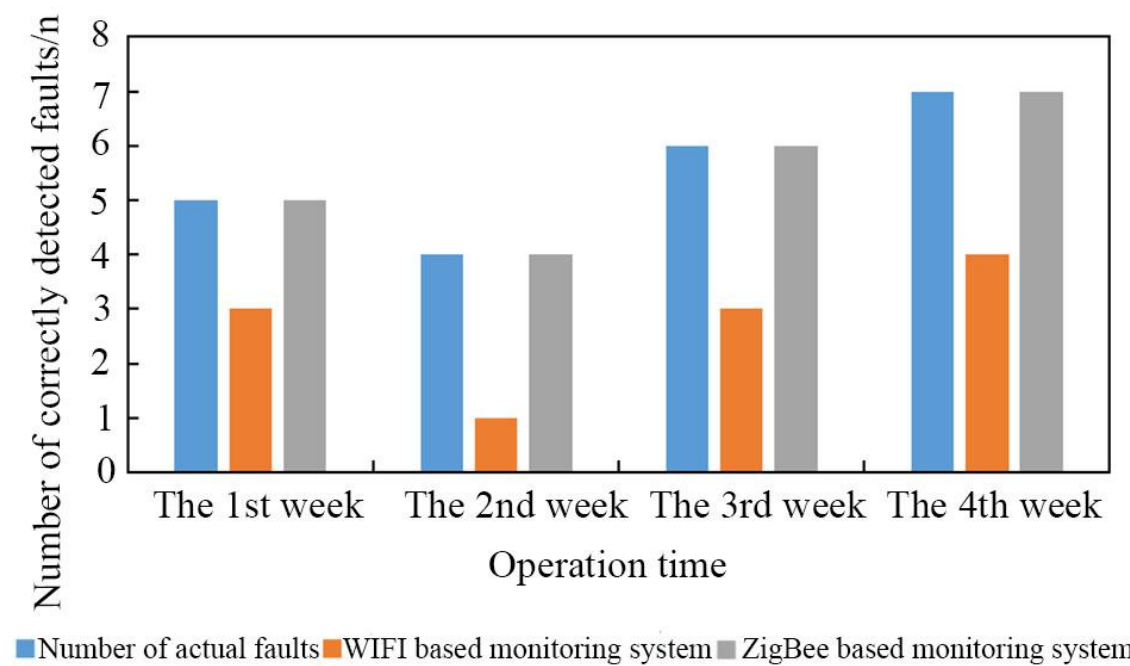

Figure 5: The Number of Correctly Detected Faults of the Power Grid Monitoring Systems under Two Wireless Communication Technologies

The relevant data of the terminal sensor and coordinator were collected by USB serial portfor packet capturing test. The packet loss rate results of the power grid monitoring systems under the two wireless communication technologies are shown in Figure 6. The average packet loss rate of the power grid monitoring system based on WiFi wireless sensor network was $1.15 \%$, and the maximum packet loss rate was $1.5 \%$; the average packet loss rate of the power grid monitoring system based on
ZigBee wireless sensor network was $0.05 \%$, and the maximum packet loss rate was $0.1 \%$. It was seen from Figure 5 that the packet loss rate of the power grid monitoring system based on ZigBee wireless sensor network was significantly lower than that based on WiFi wireless sensor network.

The reason was that ZigBee module had larger transmission range than WiFi module, and ZigBee module had stronger anti-interference ability. 


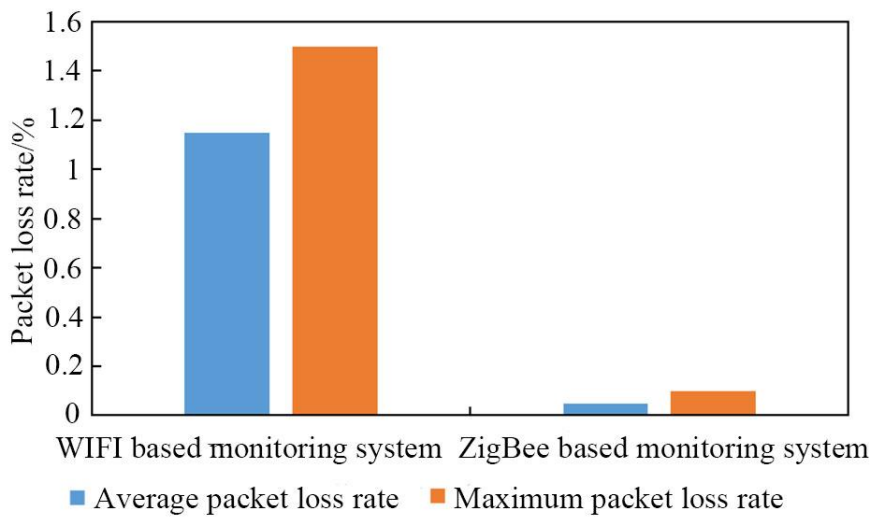

Figure 6: The Packet Loss Rate of the Power Grid Monitoring Systems under Two Wireless Communication Technologies

The time stamps of the data which were sent and received by the terminal sensor and coordinator were collected by USB serial port, and the delay of data transmission was calculated based on the time stamps. The results are shown in Figure 7.

The average time delay of the power grid monitoring system based on WiFi wireless sensor network was $120 \mathrm{~ms}$, and the maximum time delay which occurred in the sampling period was $800 \mathrm{~ms}$; the average time delay of the power grid monitoring system based on the ZigBee based wireless sensor network was $80 \mathrm{~ms}$, and the maximum time delay which occurred in the sampling period was $200 \mathrm{~ms}$.

It was seen from Figure 7 that the average and maximum delay of the power grid monitoring system based on ZigBee wireless sensor network was shorter than that using the WiFi based wireless sensor network. The reasons are as follows: firstly, the propagation of electromagnetic wave took time; secondly, when the data was lost in the wireless transmission process, the terminal sensor would send the data packet again until the coordinator accepted it successfully, and it took some time for the coordinator to receive and record the data.

Therefore, there must be a delay in data transmission in the above process.

Compared with ZigBee, the signal transmission range of WiFi module was smaller, and there was relatively frequent packet loss, resulting in many times of data retransmission and larger delay.

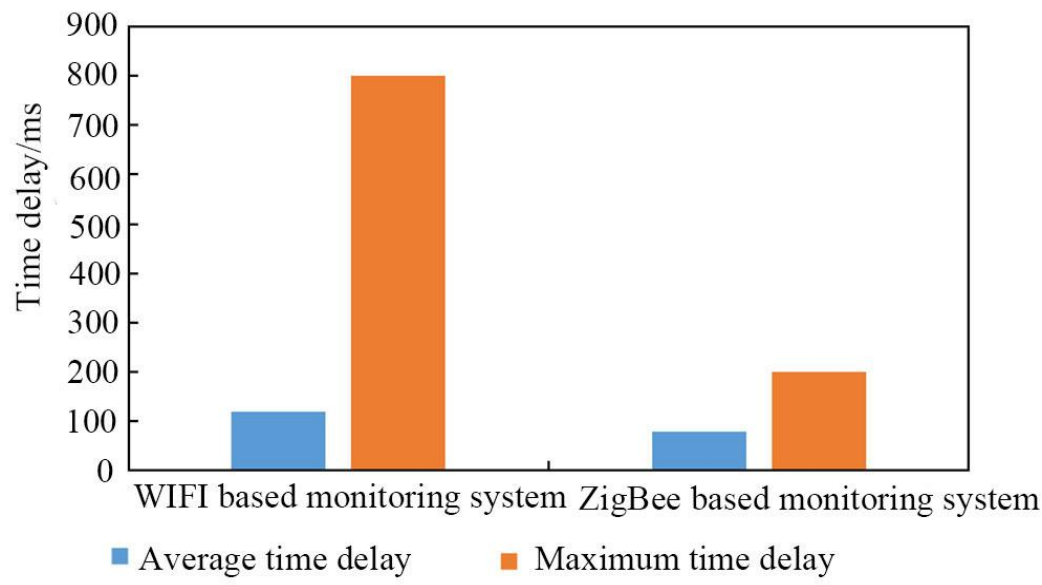

Figure 7: The Time Delay of the Power Grid Monitoring Systems under Two Wireless Communication Technologies

Limited by the space, only the actual data and monitoring data of three nodes are shown (Table 1).

The monitoring error of the power grid monitoring system under the two wireless communication technologies is shown in Figure 8.

The average error of the measurement temperature of the $\mathrm{WiFi}$ based monitoring system was $0.09^{\circ} \mathrm{C}$, the average error of the current was 0.08 $\mathrm{A}$, and the average error of the voltage was $0.09 \mathrm{~V}$.
The average error of the measurement temperature of the ZigBee based monitoring system was $0.09 \mathrm{~V}$, the average error of current was $0.02 \mathrm{~A}$, and the average error of voltage was $0.02 \mathrm{~V}$.

In the aspect of data, the measurement errors of the monitoring systems based on two wireless communication technologies were all within the acceptable range, but the comparison of the measurement errors intuitively suggested that the monitoring system based on ZigBee had smaller detection errors. 
Table 1. Actual Data and Monitoring Data of Three Nodes of Monitoring Systems under Two Wireless Communication Technologies

\begin{tabular}{|l|c|c|c|c|c|c|c|}
\hline & & $\begin{array}{c}\text { Actual } \\
\text { temperature } \\
{ }^{\circ} \mathrm{C}\end{array}$ & $\begin{array}{c}\text { Monitoring } \\
\text { temperature } \\
{ }^{\circ} \mathrm{C}\end{array}$ & $\begin{array}{c}\text { Actual } \\
\text { current } \\
\mathrm{A}\end{array}$ & $\begin{array}{c}\text { Monitorin } \\
\text { g current } \\
\mathrm{A}\end{array}$ & $\begin{array}{c}\text { Actual } \\
\text { voltage } \\
\mathrm{V}\end{array}$ & $\begin{array}{c}\text { Monitoring } \\
\text { voltage V }\end{array}$ \\
\hline $\begin{array}{l}\text { Monitoring } \\
\text { system based } \\
\text { on WiFi }\end{array}$ & Node 1 & 24.33 & 24.24 & 15.83 & 15.75 & 220.02 & 219.93 \\
\cline { 2 - 8 } & Node 2 & 24.32 & 24.41 & 15.82 & 15.90 & 220.01 & 220.10 \\
\hline \multirow{2}{*}{$\begin{array}{l}\text { Monitoring } \\
\text { system based } \\
\text { on ZigBee }\end{array}$} & Node 3 & 24.34 & 24.25 & 15.83 & 15.75 & 220.03 & 220.12 \\
\cline { 2 - 8 } & Node 2 & 24.33 & 24.30 & 15.83 & 15.81 & 220.02 & 220.00 \\
\cline { 2 - 8 } & Node 3 & 24.32 & 24.35 & 15.82 & 15.84 & 220.01 & 220.03 \\
\hline
\end{tabular}

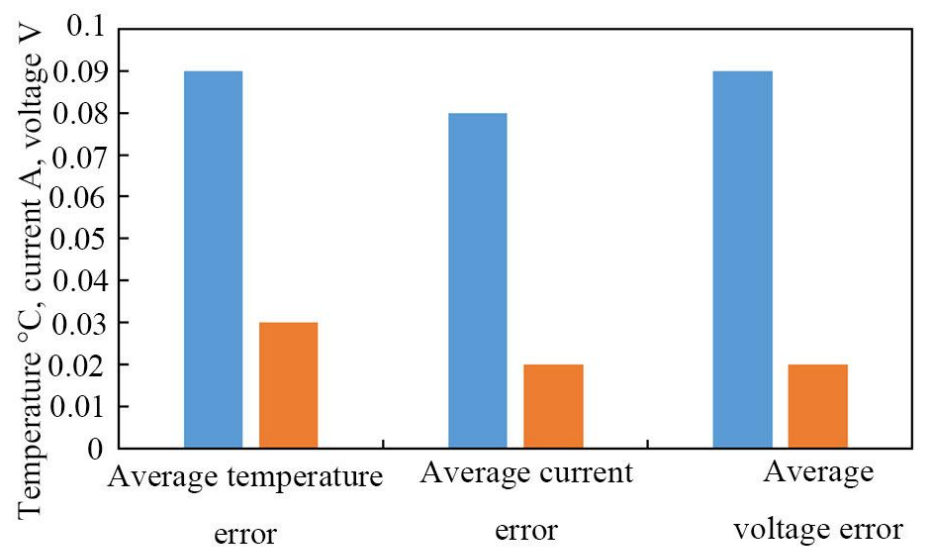

"WIFI based monitoring system $\square$ ZigBee based monitoring system

Figure 8: The Monitoring Error of Power Grid Monitoring Systems under Two Wireless Communication Technologies

\section{Conclusions}

This study briefly introduced the ZigBee based wireless communication technology and power grid monitoring system based on ZigBee wireless sensor network, tested the power grid monitoring system in a small-scale local substation, and compared it with another WiFi based power grid monitoring system. The test items included the detection accuracy of power grid fault and packet loss rate and time delay of the wireless sensor network in monitoring system.

The results are as follows: (1) in the four-week monitoring process, the power grid had 22 failures in total, and the WiFi based monitoring system used for comparison detected 11 failures correctly, while the monitoring system proposed in this study detected 22 failures correctly; (2) the wireless sensor network was tested by using USB serial port; the average packet loss rate and maximum packet loss rate of the WiFi based monitoring system were $1.15 \%$ and $1.5 \%$ respectively, and the average packet loss rate and maximum packet loss rate of the monitoring system proposed in this study were $0.05 \%$ and $0.1 \%$, respectively; ( 3 ) the average delay and maximum delay of the WiFi based monitoring system was $120 \mathrm{~ms}$ and $800 \mathrm{~ms}$ respectively, and the average delay and maximum delay of the monitoring system proposed in this study was $80 \mathrm{~ms}$ and 200 ms respectively; (4) compared with the WiFi based monitoring system, the system proposed in this study had smaller detection error for the detection of temperature, current and voltage of sensor nodes in the substation grid.

\section{References}

[1] Gao J, Asamoah K O, Sifah E B, Smahi A, Xia Q Xia H, Zhang X S, Dong G S. "GridMonitoring: Secured Sovereign Blockchain Based Monitoring on Smart Grid," IEEE Access, 2018, 6(99):99179925.

[2] Kamel T, Biletskiy Y, Chang L. "Fault diagnosis and on-line monitoring for grid-connected singlephase inverters," Electric Power Systems Research, 2015, 126:68-77.

[3] Zhou H, Wu Q, Li F, Lin W. The micro-grid monitoring software design and development based on Python language," 2015 27th Chinese Control and Decision Conference (CCDC). IEEE, 2015.

[4] Madueño G C, Nielsen J J, Kim D M, Pratas N K, Stefanović Č, Popovski P. "Assessment of LTE Wireless Access for Monitoring of Energy Distribution in the Smart Grid," IEEE Journal on 
Selected Areas in Communications, 2015, 34(3):675-688.

[5] Rekik S, Baccour N, Jmaiel M, Drira K. "Experiencing Low Power Wireless Links in Distribution Smart Grid Environments," 2018 IEEE/ACS 15th International Conference on Computer Systems and Applications (AICCSA). IEEE, 2018.

[6] Otoum S, Kantarci B, Mouftah H T. "Hierarchical trust-based black-hole detection in WSN-based smart grid monitoring," IEEE International Conference on Communications. IEEE, 2017, 7997099

[7] Grilo A, Casaca A, Nunes M, Bernardo A, Rodrigues P, Almeida J P. "A management system for low voltage grids," 2017 IEEE Manchester PowerTech. IEEE, 2017, 7980826

[8] Mouftah H T, Al-Anbagi I. "Opportunities and Challenges of Heterogeneous Networks for Substations Automation in Smart Grids," Engineering \& Technology Reference, 2015, 1(1).

[9] Zahurul S, Mariun N, Grozescu I V, Tsuyoshi H, Mitani Y, Othman ML, Hizam H, Abidin IZ. Future strategic plan analysis for integrating distributed renewable generation to smart grid through wireless sensor network: Malaysia prospect," Renewable and Sustainable Energy Reviews, 2016, 53:978-992.

[10] Nielsen J J, Ganem H, Jorguseski L, Alic K, Smolnikar M, Zhu Z, Pratas NK, Golinski M, Zhang H, Kuhar U, Fan Z, Svigelj A. Secure Real-
Time Monitoring and Management of Smart Distribution Grid using Shared Cellular Networks," IEEE Wireless Communications, 2017, 24(2):10-17.

[11] Chen K L, Chen Y R, Tsai Y P, Chen N. "A Novel Wireless Multifunctional Electronic Current Transformer Based on ZigBee-Based Communication," IEEE Transactions on Smart Grid, 2017, 8(4):1888-1897.

[12] Luo X. "Design and Implementation of Wireless Voltage Monitoring System Based on Zigbee," Chinese Journal of Electron Devices, 2017, 12(3):83-96.

[13] Sharma J, Bhattacharya P P, Jha M. "Performance Analysis of IEEE 802.15.4 in Different NLOS Environments of Smart Grids," International Journal of Sensors Wireless Communications \& Control, 2016, 6(3):216220.

[14] Ortega A, Schweitzer C M, Shinoda A A, Ortega A V. "Simulation of the DNP3 protocol over TCP/IP on a network IEEE 802.11g ad-hoc with smart meter," 2016 IEEE Andescon. IEEE, 2016, 7836213

[15] Ortega A, Shinoda A A, Schweitzer C M, Granelli $\mathrm{F}$, Ortega A V, Bonvecchio F. "Proposal DNP3 protocol simulation on NS-2 in IEEE 802.11g wireless network ad hoc over TCP/IP in smart grid applications," 2015 IEEE PES Innovative Smart Grid Technologies Latin America (ISGT LATAM). IEEE, 2015, 7381229 